\title{
Blunted Neural Reward Responsiveness and Recent Suicidal Ideation in Children and Adolescents: Failure to Replicate Across Two Independent Samples
}

Austin J. Gallyer, M.S. ${ }^{1}$, Kreshnik Burani, M.S. ${ }^{1}$, Elizabeth M. Mulligan, M.S. ${ }^{1}$, Nicholas Santopetro, B.S. ${ }^{1}$, Sean P. Dougherty, M.S. ${ }^{1}$, Michelle E. Jeon, M.A. ${ }^{1}$, Brady D. Nelson, Ph.D. ${ }^{2}$, Thomas E. Joiner, Ph.D. ${ }^{1}$, \& Greg Hajcak, Ph.D. ${ }^{1,3}$ ${ }^{1}$ Department of Psychology, Florida State University

${ }^{2}$ Department of Psychology, Stony Brook University ${ }^{3}$ Department of Biomedical Sciences, Florida State University

\section{Author Note}

This work was supported by grants from the National Institute of Mental Health (T32MH093311; R01MH106477; R01MH097767). This work was also supported in part by the Military Suicide Research Consortium (MSRC), an effort supported by the Assistant Secretary of Defense for Health Affairs under Award No. (W81XWH-16-2-0004). Opinions, interpretations, conclusions, and recommendations are those of the author and are not necessarily endorsed by the MSRC or the Department of Defense.

Correspondence concerning this article should be addressed to Austin J. Gallyer, M.S., 1107 West Call St., Tallahassee, FL, 32306. Email: agallyer@neuro.fsu.edu. 


\begin{abstract}
A recent study by Tsypes, Owens, and Gibb (2019) found that children with recent suicidal ideation had blunted neural reward processing, as measured by the reward positivity (RewP), compared to matched controls, and that this difference was driven by reduced neural responses to monetary loss, rather than blunted neural response to monetary reward. Here, we aimed to conceptually replicate and extend these findings in two large samples of children and adolescents ( $n=275$ and $n=235$ ). Results from our conceptual replication found no evidence that children and adolescents with suicidal ideation have abnormal reward or loss processing. We extended these findings in a longitudinal sample of children and adolescents with two time points and found no evidence that reward- or loss-related ERPs predict changes in suicidal ideation. The results highlight the need for greater statistical power, and continued research examining the neural underpinnings of suicidal thoughts and behaviors.

Keywords: suicidal ideation, rewards, ERP, children, reward positivity, RewP, feedback negativity, suicide
\end{abstract}




\section{Blunted Neural Reward Responsiveness and Recent Suicidal Ideation in Children and Adolescents: Efforts to Conceptually Replicate in Two Large Samples}

Suicide is a growing problem among children and adolescents. In the United States from 1999 to 2018, the suicide rate increased from 1.2 to 2.9, and from 8.0 to 11.4 , per 100,000 for those 10-14 and 15-19 years old, respectively (National Center for Health Statistics, 2018). Moreover, suicide is the second leading cause of death for children and adolescents between 10 and 19 years of age (Centers for Disease Control and Prevention, 2018). However, despite over 50 years of research, the field's ability to predict who will die by suicide remains suboptimal (Franklin et al., 2017). It has been suggested that this lack of predictive power may be due to failures to examine risk factors for suicide from a transdiagnostic perspective (Glenn et al., 2017). In particular, the Research Domain Criteria (RDoC), a framework developed by the National Institute of Mental Health that aims to integrate multiple levels of analysis to identify the underlying neural mechanisms of psychopathology, has been proposed as a way to improve the prediction and understanding of suicidal thoughts and behaviors (Glenn et al., 2017; Insel et al., 2010).

One way to apply RDoC to the understanding of suicidal thoughts and behaviors is through the use of event-related potentials (ERPs). ERPs are direct measures of neural response to specific events (e.g., onset of feedback) recorded using the electroencephalogram. ERPs can be used as indicators of risk for, and correlates of, psychopathology, and as biomarkers for psychiatric disorders (Hajcak et al., 2019). In this vein, ERPs have been suggested as a way to improve the prediction of suicidal thoughts and behaviors (Gibb \& Tsypes, 2019).

The reward positivity (RewP) is an ERP that indexes the neural response to reward receipt, typically measured as the difference between monetary rewards and losses (Proudfit, 2015). The RewP is related to self-report and behavioral measures of reward processing 
(Bress \& Hajcak, 2013; Pizzagalli et al., 2005), and is correlated with activation in rewardrelated brain regions, including the ventral striatum and the medial prefrontal cortices (Carlson et al., 2011). A blunted RewP is correlated with increased depressive symptoms and predicts the first onset of major depressive disorder (Belden et al., 2016; Bress et al., 2012, 2013; Klawohn et al., 2020; Nelson et al., 2016).

Critically, recent work suggests that the RewP may have utility for the study of SI. Tsypes, Owens, and Gibb (2019) found that children with recent SI had a reduced RewP, compared to demographically and clinically matched controls. Moreover, the authors found that this difference was driven by an enhanced (i.e., more positive) neural response to losses. These results suggest that children with SI have blunted reward processing, compared to children without SI, implying that differences in the RewP may be useful in identifying children with SI. Tsypes and colleagues’ findings are important for their potential to aid in identifying children at risk. Their results also point specifically to neural responses to losses as a marker of suicidal ideation among this population. Indeed, the primary goal of the present study was to conceptually replicate these findings in two samples of children and adolescents.

We examined whether the RewP was blunted in children and adolescents with recent SI, compared to controls, using similar methods, measures, and processing procedures as those used by Tsypes and colleagues (see Methods) in two large samples. Our sample size is especially important, given that the study by Types and colleagues, as in many ERP studies, may have been underpowered (Clayson et al., 2019). Specifically, the study by Tsypes and colleagues found a relatively large difference (Hedges' $g=.60$ ) in the RewP between children with SI and controls. However, a recent meta-analysis estimated that the average difference in ERPs between those with SI and controls is smaller, with a Hedges' $g=.27$ (Gallyer et al., 2020, preprint under review). Low power not only decreases the probability of detecting 
small true effects, but also increases the probability of false positives (Button et al., 2013). While the meta-analysis by Gallyer and colleages was not specific to the RewP, it is a reasonable estimate of the expected difference in the RewP between those with SI and controls. Moreover, we sought to expand on Tsypes and colleagues' findings by examining whether the RewP is not only related to SI cross-sectionally, but also whether it could predict various SI trajectories over time.

In our first sample, consistent with Tsypes et al. (2019), we hypothesized that children and adolescents with recent SI would have a significantly smaller RewP than those without recent SI and that this effect would be driven by a blunted neural response to loss trials. For our second sample, we hypothesized the following: (1) Children and adolescents with recent SI would have a significantly smaller RewP than those without recent SI cross-sectionally at Time 1 and at Time 2; and (2) children and adolescents with SI at both time points would have significantly smaller RewP across both time points than those with SI at just one time point and those who did not experience SI at either time point.

\section{Study 1: Conceptual Replication}

\section{Methods}

\section{Participants}

Our first sample consisted of 275 children and adolescents. Participants ranged from 11.01 to 14.98 years of age $(M=12.91, S D=1.14)$. Our sample consisted of slightly more males than females (54.9\% male). In this sample, $72.36 \%$ identified as white, $10.18 \%$ identified as African American, 6.18\% identified as Hispanic, 4.73\% identified as Asian, and 6.55\% identified their race/ethnicity as “other.” Notably, our sample’s racial and gender demographics was similar to Tsypes and colleagues’ (2019) sample, but our sample was slightly older. Based on the present study’s sample size and an alpha of .05, we were powered at .84 to detect an effect size of Hedges' $g=.60$ (calculation based on information in Tsypes 
et al. [2019]) for our difference in the RewP between those with recent SI and controls. As noted above, however, there is a possibility that the true effect is closer to Hedges’ $g=.27$ (Gallyer et al., 2020, preprint under review). If so, then we were only powered at .26 to detect this effect size. Participants and their parents provided informed consent and assent. The study was approved by the Institutional Review Broad at Florida State University.

\section{Measures}

Suicidal Ideation Measure. To assess for the presence of recent SI (i.e., within the past two weeks), we used item $9^{1}$ of the original Children's Depression Inventory (CDI; Kovacs, 1981) and the Schedule for School-Age Children-Present and Lifetime Version (KSADS-PL; Kaufman et al., 1997). Specifically, in line with Tsypes and colleagues (2019), during the K-SADS-PL interview the children and their parents were asked "Sometimes children who get upset or feel bad think about dying or even killing themselves. Have you/your child ever had these types of thoughts?” The individuals who endorsed a " 1 " or "2" and/or the presence of SI within the past two weeks on the K-SADS-PL interview were classified as having recent SI. Using this approach, 27 participants endorsed having recent SI.

Doors Task. To measure neural responses to reward, we used the same monetary guessing task used by Tsypes and colleagues (2019). The doors task is frequently used in ERP studies of reward processing (Bress et al., 2012, 2013; Burani et al., 2019; Klawohn et al., 2020). During this task, participants are presented with two doors and instructed to guess which door has the monetary prize behind it. To make their selection, the participants use left and right mouse buttons corresponding with the door on the left and right, respectively. After selecting a door, visual feedback is given consisting of either a green arrow pointing upward, indicating that the participant won $\$ 0.50$, or a red arrow pointing downward, indicating that the participant lost $\$ 0.25$. Feedback was presented for $2,000 \mathrm{~ms}$; then the message “Click for

1 Note that we used the original CDI, but that in the revised version, the suicide item is item 8. 
next round” was presented. Our task consisted of 60 trials presented in two blocks of 30 trials. For this task, 30 win and 30 loss trials were presented in random order.

ERP Data Collection and Processing. During the doors task, electroencephalogram (EEG) was recorded with an active electrode EEG-system (ActiCHamp, Brain Products $\mathrm{GmbH}$ ) with 32 electrodes positioned in accordance with the 10/20-system (ActiCAP, Brain Products $\mathrm{GmbH})$. Electrode $\mathrm{Cz}$ served as the online reference, a ground electrode was placed on the forehead, and two electrodes were placed on the mastoids. Electrooculogram was recorded from four additional electrodes: two $1 \mathrm{~cm}$ above and below the left eye and two at the outer canthi of both eyes. Continuous EEG waves were recorded at a sampling rate of $1000 \mathrm{~Hz}$ using a bandpass recording filter of 0.01-100 Hz. EEG data were processed using Brain Vision Analyzer, Version 2.1 (Brain Products, Gilching, Germany). For some participants $(n=14)$, at least one channel had to be interpolated using surrounding channels. Then, for all participants, data were re-referenced to the average of the mastoid electrodes and filtered from 0.01 to $30 \mathrm{~Hz}$ (Butterworth, $4^{\text {th }}$ order). We then extracted 1000 ms feedbacklocked epochs, starting 200 ms before feedback presentation. Next, we corrected for eye movement artifacts using the algorithm developed by Gratton, Coles, and Donchin (1983). After this correction, segments that contained voltage steps $>50 \mathrm{mV}$ between sample points, a voltage difference of $175 \mathrm{mV}$ within a 400-ms interval, or a maximum voltage difference of $<0.5 \mathrm{mV}$ within 100-ms intervals were automatically rejected.

Win and loss trials were averaged separately, and a baseline correction was applied using the 200-ms preceding feedback onset. Then, in line with Tsypes and colleagues' (2019) procedures, we exported these averages for temporospatial principal component analysis (PCA), a factor analytic approach used to parse the ERP waveform into underlying constituent components. PCA was conducted using the ERP PCA Toolkit (Version 2.82; Dien, 2010a). In line with evidence-based recommendations (Dien, 2010b), we first conducted a 
temporal PCA using promax rotation. Covariance matrix and Kaiser normalization were used for this PCA (Dien et al., 2005). Based on the resulting scree plot, 15 factors were extracted in the temporal domain. We then analyzed the spatial distributions of these temporal factors by conducting a spatial PCA using infomax rotation (Dien, 2010b). Based on the averaged scree plot for all 15 temporal factors, we extracted four spatial factors, yielding 60 factor combinations. Twenty factors each accounted for more than $0.50 \%$ of the variance and were retained for further inspection (Kaiser, 1960). After examining the factors, TF03SF1 (i.e., temporal factor 3 , spatial factor 1 ), which accounted for $11.20 \%$ of the variance, most closely resembled the RewP: this factor was maximal at the Cz electrode site at $235 \mathrm{~ms}$ following feedback onset and was more positive following win than loss trials.

\section{Data Analytic Plan}

All statistical analyses were conducted in R (Version 3.5.2; R Core Team, 2018), using the following packages: “tidyverse” (Version 1.3.0; Wickham et al., 2019), "haven” (Version 2.2.0; Wickham \& Miller, 2019), “afex” (Version 0.23-0; Singmann et al., 2019), “here” (Version 0.1; Müller, 2017), “DataExplorer” (Version 0.7.0; Cui, 2018), “emmeans” (Version 1.3.2; Lenth, 2019), and “psych” (Version 1.8.4; Revelle, 2018). To test our hypotheses, we conducted a 2x2 (condition: win, loss; ideation: no SI, recent SI) mixedmeasures ANOVA. We also conducted a one-way between-subjects (ideation: no SI, recent SI) ANOVA with the $\Delta$ RewP factor score (factor score wins minus factor score losses) as the dependent variable². Finally, we reran both of these ANOVAs with age as a covariate.

\section{Results}

The means and standard deviations of study variables across Study 1 and Study 2, are presented in Table 1. The mixed-measures ANOVA revealed an independent effect of condition $\left(F[1,262]=45.61, p<.001, \eta^{2}=.024\right)$, such that wins $(M=17.9,95 \%$ CI $[16.80$, temporospatial PCA. The pattern of results remained the same. 
19.00]) were greater than losses $(M=13.80,95 \%$ CI $[12.80,14.9])$. There was no independent effect of ideation $\left(F[1,262]=1.66, p=.200, \eta^{2}=.005\right)$ or interaction between ideation and condition $\left(F[1,262]=0.08, p=.773, \eta^{2}<.001\right)$. Our ANCOVA with age as a covariate revealed no statistically significant effects, including the interaction between ideation and condition $\left(F[1,260]=0.28, p=0.601, \eta^{2}<.001\right)$. Our between-subjects ANOVA also failed to find a main effect of ideation $\left(F[1,262]=0.08, p=.773, \eta^{2}<.001\right.$; see Figure 1). As with our first ANOVA, adding age as a covariate to this analysis did not reveal any significant effects, including the main effect of ideation $(F[1,260]=0.28, p=.601), \eta^{2}$ $=.001)$ and the interaction between age and ideation $\left(F[1,260]=0.25, p=0.621, \eta^{2}<.001\right)$.

\section{Study 2: Extension}

\section{Method}

\section{Participants}

Our second sample consisted of 325 child and adolescent females who ranged from 8.01 to 15.04 years of age $(M=12.42, S D=1.80)$ at Time 1 . At the two-year follow-up, the sample consisted of 235 participants $(M=14.41, S D=1.82)$. Notably, there were no differences in race $\left(\mathrm{X}^{2}[12]=4.85, p=.963\right)$ between those who dropped out of the study and those who returned at Time 1, but those who dropped out of the study were on average 0.71 years older than those who remained in the study $(t[129.77]=3.09, p=.013)$. In this sample, 81.54\% identified their race as white/Caucasian, 5.85\% identified as Black/African American, 0.31\% identified as Native Hawaiian/Pacific Islander, 0.31\% identified as American Indian/Alaskan Native, and 6.15\% identified their race as “other.” As in Study 1, this racial breakdown is similar to the sample in Tsypes and colleagues (2019), and our sample was slightly older than the participants recruited by Tsypes and colleagues. Participants were recruited from the community through a commercial mailing list, using flyers, and using word-of-mouth. All participants and their parents provided informed consent 
and assent, as approved by the Institutional Review Board at Stony Brook University.

\section{Measures}

Suicidal Ideation Measure. As in Study 1, we used item 9 of the CDI and the KSADS-PL interview to assess recent SI, and participants who endorsed above "0" and/or endorsed SI during the K-SADS-PL were categorized as having recent SI. Based on these variables at both time points, we created another variable to represent participants' SI at both time points. The coding was: “0” for not endorsing SI at either time point; “ 1 ” for endorsing SI at Time 1 but not at Time 2; “2” for not endorsing SI at Time 1 but endorsing SI at Time 2; and " 3 ” for endorsing SI at both time points. Based on this approach, 185 were categorized as “No ideation,” nineteen as Time 1 SI only, sixteen as Time 2 SI only, and fourteen with recent SI at Time 1 and Time 2.

Doors Task. We used the same doors task that was used in Study 1.

ERP Data Collection and Processing. During the doors task, continuous EEG was recorded using the ActiveTwo BioSemi system (BioSemi, Amsterdam, Netherlands) with a cap containing 34 electrodes placed according to the 10/20 system (i.e., 32 channels plus FCz and Iz). We also placed electrodes above and below the left eye, as well as near the outer canthi of both eyes, to measure vertical and horizontal electrooculographic activity, respectively. Two electrodes were also placed on the left and right mastoids. The EEG signal was preamplified at the electrode, and data were digitized at a 24-bit resolution with a sampling rate of $1024 \mathrm{~Hz}$ using a low-pass fifth-order sinc filter with a half-power cutoff of $204 \mathrm{~Hz}$. Active electrodes were measured online with reference to a Common Mode Sense active electrode constructing a monopolar channel.

Data were processed using BrainVision Analyzer, Version 2.1 (Brain Products, Gliching, Germany). The data processing procedures were largely the same as in Study 1, with small differences in artifact detection: Segments that contained voltage steps $>50 \mathrm{mV}$ 
between sample points, a voltage difference of $300 \mathrm{mV}$ within a segment, or a maximum voltage difference of $<0.5 \mathrm{mV}$ within 100 -ms intervals were automatically rejected. As in Study 1, we conducted temporospatial PCA using ERP PCA Toolkit (Dien, 2010a). To do so, we first conducted a temporal PCA with promax rotation. A covariance matrix and Kaiser normalization were used for this PCA (Dien et al., 2005). Based on the resulting scree plot, 22 temporal factors were extracted in the temporal domain. We then analyzed the spatial distributions of these temporal factors by conducting a spatial PCA using infomax rotation (Dien, 2010b). Based on the averaged scree plot for all 22 temporal factors, we extracted 4 spatial factors, yielding 88 factor combinations. Twenty-four factors each accounted for more than $0.50 \%$ of the variance and were retained for further inspection (Kaiser, 1960). TF07SF1, which accounted for $0.8 \%$ of the variance, most closely resembled the RewP, as it was maximal at the FCz electrode site at $346 \mathrm{~ms}$ and more positive for win trials than for loss trials. Thus, factor scores from this PCA factor were used as the RewP measure for Wave 1.

We repeated this same process for Wave 2. Our temporal PCA resulted in 19 factors, and our spatial PCA resulted in 3 factors, yielding 57 factor combinations together. Eighteen factors each accounted for more than $0.50 \%$ of the variance and were retained for further inspection (Kaiser, 1960). The TF06SF1 factor, which accounted for 1.5\% of the variance, most closely resembled the RewP, as it was maximal at the FCz electrode site at 278 ms and more positive for win trials than for loss trials.

\section{Data Analytic Plan}

We used the same statistical software and packages that were used in Study 1. To test our hypotheses that (1) children and adolescents with recent SI would have a significantly smaller RewP than those without recent SI cross-sectionally at Time 1 and Time 2 and (2) children and adolescents with SI at both time points would have significantly smaller RewP across both time points than those with SI at just one or neither time points, we conducted a 2 
(time: Wave 1, Wave 2) x 2 (condition: win, loss) x 4 (ideation history: no SI, SI at Wave 1 only, SI at Wave 2 only, SI at Wave 1 and Wave 2) mixed-measures ANOVA, with mean mV as the dependent variable. We conducted a 2 (time: Wave 1, Wave 2) x 4 (ideation history: no SI, SI at Wave 1 only, SI at Wave 2 only, SI at Wave 1 and Wave 2) ANOVA using the factor score $\Delta$ RewP (factor score wins minus factor score losses) as the dependent variable. ${ }^{3}$ We also reran both of these analyses using age as a covariate.

\section{Results}

As expected, we found a main effect of condition $\left(F[1,249]=55.88, p<.001, \eta^{2}\right.$ $=.039)$, such that wins $(M=4.98,95 \%$ CI $[4.28,5.68])$ were greater than losses $(M=0.95$, $95 \%$ CI $[0.24,1.65])$. We also found a main effect of time $\left(F[1,249]=14.76, p<.001, \eta^{2}\right.$ $=.020)$, such that Wave 1 factor scores $(M=1.53$, 95\% CI $[0.67,2.40])$ were less positive than Wave $2(M=4.39,95 \%$ CI $[3.53,5.26])$. Contrary to our hypothesis, we did not find a main effect of ideation history $\left(F[3,249]=0.90, p=.443, \eta^{2}=.004\right)$, nor did we find a significant three-way interaction among ideation history, time, and condition $(F[3,249]=$ 0.90, $\left.p=0.444, \eta^{2}=.001\right)$. We also did not find a two-way interaction between ideation history and condition $\left(F[3,249]=1.45, p=.230, \eta^{2}=.003\right)$. Rerunning this analysis with age as a covariate did not reveal a three-way interaction among ideation history, time, and condition $\left(F[3,245]=1.17, p=.322, \eta^{2}=.001\right)$, nor was there a two-way interaction between ideation history and condition $\left(F[3,245]=0.18, p=.911, \eta^{2}<.001\right)$.

For our second mixed-measures ANOVA, we similarly did not find a main effect of ideation history $\left(F[1,251]=0.10, p=.751, \eta^{2}<.001\right.$; see Figure 2$)$ or time $(F[1,251]=$ $\left.0.08, p=.777, \eta^{2}<.001\right)$ or a two-way interaction between ideation history and time $(F[1$, $\left.251]=0.02, p=.890, \eta^{2}<.001\right)$. Rerunning this analysis with age as a covariate similarly

3 As in study 1, we reran these analyses using the area RewP measure (250-350 ms) without using PCA. Results remained the same. We also ran these analyses collapsing those who had recent SI at one time point into one group. Results remained the same. 
did not reveal a main effect of ideation history $\left(F[1,249]=0.12, p=.730, \eta^{2}<.001\right)$, neither was there a two-way interaction between ideation history and time $(F[1,249]=2.23, p$ $\left.=.136, \eta^{2}=.003\right)$.

\section{Discussion}

The purpose of this study was to test whether children and adolescents with recent suicidal ideation (SI) have blunted neural response to gains and losses cross-sectionally in a large sample of males and females, and longitudinally in a sample of females. A previous cross-sectional study found that children with SI exhibit aberrant reward processing compared to matched controls, and this difference appeared to be driven by neural responses to loss trials, rather than gain trials (Tsypes et al., 2019). However, in our conceptual replication using nearly identical methods, we did not find any evidence that children and adolescents with recent SI had different neural reward responses than those without recent SI.

We extended these results by examining reward processing and SI in one of the samples that also had longitudinal data. Here, we again did not find any differences in neural response to reward between children and adolescents with recent SI and those without recent SI. Moreover, we found no evidence of differences in neural response to reward at either time point between children and adolescents showing various patterns of recent SI (i.e., SI at Time 1 only, SI at Time 2 only, and SI at Time 1 and Time 2) and those without recent SI. Together, our results suggest that children and adolescents with recent SI do not have deficits in neural systems responsible for reward processing as indexed by the RewP.

There are at least four reasons why our results do not align with the study by Tsypes and colleagues (2019). First, the study by Tsypes and colleagues could have been a false positive. Indeed, though Tsypes and colleagues found a significant effect (i.e., $p=.02$ ), it is important to remember that this $p$ value is model- and sample-specific and that it does not 
provide the probability that the null hypothesis is true ${ }^{4}$ (see Greenland et al., 2016). The second possibility is that there is a true effect but that this effect is much smaller than even our larger sample sizes could reliably detect. A recent meta-analysis of the relation between all ERPs and SI found an average Hedges' $g=0.27$, an effect size that is unlikely to be detected in samples with fewer than 100 participants (Gallyer et al., 2020, preprint under review). Though our samples were well-powered to detect the effect size found by Tsypes and colleagues (i.e., Hedges’ $g=$.60; 2019), much larger samples (i.e., over 200 per group) would be needed if a true effect exists that is closer to Hedges' $g=0.27$. Third, our results could be a false negative, or type II error, despite being well-powered (i.e., if the true effect is Hedges’ $g=.60$ ) to detect an effect. Indeed, mixed evidence across multiple studies is to be expected for true effects if studies are powered at .80 (Lakens \& Etz, 2017). Fourth, though we aimed to replicate the methods and sample used by Tsypes and colleagues as closely as possible, our contrasting results could have been due to differences in our samples. For example, both of our samples consisted of individuals who were slightly older, which may have impacted our findings. Importantly, though, adding age as a covariate to our analyses did not change our results. Similarly, because Tsypes and colleagues study used PCA, it is difficult, if not impossible, to exactly replicate the weightings of time and space in the quantification of the RewP. Moreove, though we chose the PCA factors that most closely represented the RewP in each our samples, our Study 1 PCA factor and Study 2 Wave 1 factor peaked at slightly different times than is traditionally attributed to the RewP (i.e., 235 ms and 346 ms vs. 250-350). Therefore, it is possible that factor selection influenced our results. Importantly, however, we found null results across Study 1 and Study 2 even if we used area measures (250-350 ms) without conducting PCA. Therefore, our results appear to be robust to other methodological choices.

4 We want to be clear that this is a common misconception, but that Tsypes and colleagues did not make this assertion. 
The current study has notable strengths. First, our study largely used the same methods as the study we attempted to conceptually replicate, including the same task, measure of recent SI, and similar data processing and data analytic procedures. The present study also used relatively large samples that are not typically seen in ERP research, making our study well-powered to detect medium-to-large effects. Our study also used two independent samples and extended on previous work by using an additional longitudinal sample, with EEG and SI measurements at both time points. As such, the current study is the first to our knowledge to attempt to prospectively predict SI using an ERP-here, the RewP.

Despite these strengths, the current study also has some limitations. First, though our study was powered to detect the effect size previously found by Tsypes and colleagues, much larger samples are needed to detect smaller effects. Due to measurement error, it is expected that ERPs will have small-to-moderate effects with behavioral and self-report outcomes. Given the low base rate of SI and other suicidal outcomes, large samples are crucial for elucidating the relationship between the RewP and suicidal thoughts and behaviors. Second, our second sample consisted only of female participants. Though this potential demographic limitation is mitigated by our first sample, which has a fairly even split of males and females, this fact underscores that our longitudinal analyses may not necessarily apply to other samples that include males.

\section{Conclusions}

In summary, the present study attempted to conceptually replicate and extend on previous findings that children with recent suicidal ideation had blunted neural reward processing, compared to controls. In two samples, we did not find evidence of this effect, and also did not find evidence that blunted reward processing was associated with future suicidal ideation. The current results highlight the need for larger sample sizes and multiple studies when investigating the relationship between neural reward processing and suicidal ideation. 
Our hope is that the present results will spur further research into the neural underpinnings of suicidal thoughts and behaviors and that greater collaboration and larger sample sizes will begin to dominate this field. Such approaches will greatly enhance our ability to contribute to the knowledge of the neurophysiology of suicidal thoughts and behaviors and will allow us to explore the potential clinical applications of event-related potentials to suicidal thoughts and behaviors. 


\section{References}

Belden, A. C., Irvin, K., Hajcak, G., Kappenman, E. S., Kelly, D., Karlow, S., Luby, J. L., \& Barch, D. M. (2016). Neural correlates of reward processing in depressed and healthy preschool-age children. Journal of the American Academy of Child \& Adolescent Psychiatry, 55(12), 1081-1089.

Bress, J. N., Foti, D., Kotov, R., Klein, D. N., \& Hajcak, G. (2013). Blunted neural response to rewards prospectively predicts depression in adolescent girls: Feedback negativity predicts depression. Psychophysiology, 50(1), 74-81. https://doi.org/10.1111/j.14698986.2012.01485.x

Bress, J. N., \& Hajcak, G. (2013). Self-report and behavioral measures of reward sensitivity predict the feedback negativity: Feedback negativity, self-report, and behavior. Psychophysiology, 50(7), 610-616. https://doi.org/10.1111/psyp.12053

Bress, J. N., Smith, E., Foti, D., Klein, D. N., \& Hajcak, G. (2012). Neural response to reward and depressive symptoms in late childhood to early adolescence. Biological Psychology, 89(1), 156-162. https://doi.org/10.1016/j.biopsycho.2011.10.004

Burani, K., Mulligan, E. M., Klawohn, J., Luking, K. R., Nelson, B. D., \& Hajcak, G. (2019). Longitudinal increases in reward-related neural activity in early adolescence: Evidence from event-related potentials (ERPs). Developmental Cognitive Neuroscience, 36, 100620. https://doi.org/10.1016/j.dcn.2019.100620

Button, K. S., Ioannidis, J. P. A., Mokrysz, C., Nosek, B. A., Flint, J., Robinson, E. S. J., \& Munafò, M. R. (2013). Power failure: Why small sample size undermines the reliability of neuroscience. Nature Reviews Neuroscience, 14. https://doi.org/10.1038/ nrn3475

Carlson, J. M., Foti, D., Mujica-Parodi, L. R., Harmon-Jones, E., \& Hajcak, G. (2011). Ventral striatal and medial prefrontal BOLD activation is correlated with rewardrelated electrocortical activity: A combined ERP and fMRI study. NeuroImage, 57(4), 1608-1616. https://doi.org/10.1016/j.neuroimage.2011.05.037

Centers for Disease Control. (2018). WISQARS: Web-Based Injury Statistics Query and 
Reporting System [Government Database]. www.cdc.gov/injury/wisqars

Clayson, P. E., Carbine, K. A., Baldwin, S. A., \& Larson, M. J. (2019). Methodological reporting behavior, sample sizes, and statistical power in studies of event related potentials: Barriers to reproducibility and replicability. Psychophysiology. https://doi.org/10.1111/psyp.13437

Cui, B. (2018). DataExplorer: Data explorer. https://CRAN.R-project.org/package=DataExplorer

Dien, J. (2010a). Evaluating two-step PCA of ERP data with Geomin, Infomax, Oblimin, Promax, and Varimax rotations. Psychophysiology, 47(1), 170-183. https://doi.org/10.1111/j.1469-8986.2009.00885.x

Dien, J. (2010b). The ERP PCA Toolkit: An open source program for advanced statistical analysis of event-related potential data. Journal of Neuroscience Methods, 187(1), 138-145. https://doi.org/10.1016/j.jneumeth.2009.12.009

Dien, J., Beal, D. J., \& Berg, P. (2005). Optimizing principal components analysis of eventrelated potentials: Matrix type, factor loading weighting, extraction, and rotations. Clinical Neurophysiology, 116(8), 1808-1825. https://doi.org/10.1016/j.clinph.2004.11.025

Franklin, J. C., Ribeiro, J. D., Fox, K. R., Bentley, K. H., Kleiman, E. M., Huang, X., Musacchio, K. M., Jaroszewski, A. C., Chang, B. P., \& Nock, M. K. (2017). Risk factors for suicidal thoughts and behaviors: A meta-analysis of 50 years of research. Psychological Bulletin, 143(2), 187-232. https://doi.org/10.1037/bul0000084

Gallyer, A. J., Dougherty, S. P., Burani, K., Albanese, B. J., Joiner, T. E., \& Hajcak, G. (2020). Suicidal thoughts, behaviors, and event-related potentials: A meta-analysis. BioRxiv. https://doi.org/10.1101/2020.04.29.069005

Gibb, B. E., \& Tsypes, A. (2019). Using Event-Related Potentials to Improve Our Prediction of Suicide Risk. Biological Psychiatry: Cognitive Neuroscience and Neuroimaging, 4(10), 854-855. https://doi.org/10.1016/j.bpsc.2019.08.003

Glenn, C. R., Cha, C. B., Kleiman, E. M., \& Nock, M. K. (2017). Understanding suicide risk 
within the research domain criteria (RDoC) framework: Insights, challenges, and future research considerations. Clinical Psychological Science, 5(3), 568-592.

Gratton, G., Coles, M. G. H., \& Donchin, E. (1983). A new method for off-line removal of ocular artifact. Electroencephalography and Clinical Neurophysiology, 55, 468-484.

Greenland, S., Senn, S. J., Rothman, K. J., Carlin, J. B., Poole, C., Goodman, S. N., \& Altman, D. G. (2016). Statistical tests, P values, confidence intervals, and power: A guide to misinterpretations. European Journal of Epidemiology, 31(4), 337-350. https://doi.org/10.1007/s10654-016-0149-3

Hajcak, G., Klawohn, J., \& Meyer, A. (2019). The Utility of Event-Related Potentials in Clinical Psychology. Annual Review of Clinical Psychology, 15(1), 71-95. https://doi.org/10.1146/annurev-clinpsy-050718-095457

Insel, T., Cuthbert, B., Garvey, M., Heinssen, R., Pine, D. S., Quinn, K., Sanislow, C., \& Wang, P. (2010). Research domain criteria (RDoC): Toward a new classification framework for research on mental disorders. American Journal of Psychiatry, 167(7), 748-751.

Kaiser, H. F. (1960). The Application of Electronic Computers to Factor Analysis. Educational and Psychological Measurement, 20(1), 141-151. https://doi.org/10.1177/001316446002000116

Kaufman, J., Birmaher, B., Brent, D., Rao, U., Flynn, C., Moreci, P., Williamson, D., \& Ryan, N. (1997). Schedule for affective disorders and schizophrenia for school-age childrenpresent and lifetime version (K-SADS-PL): Initial reliability and validity data. Journal of the American Academy of Child \& Adolescent Psychiatry, 36(7), 980-988.

Klawohn, J., Burani, K., Bruchnak, A., Santopetro, N., \& Hajcak, G. (2020). Reduced neural response to reward and pleasant pictures independently relate to depression. Psychological Medicine, 1-9. https://doi.org/10.1017/S0033291719003659

Lakens, D., \& Etz, A. J. (2017). Too True to be Bad: When Sets of Studies With Significant and Nonsignificant Findings Are Probably True. Social Psychological and Personality Science, 8(8), 875-881. https://doi.org/10.1177/1948550617693058 
Lenth, R. (2019). emmeans: Estimated marginal means, aka least-squares means. https://CRAN.R-project.org/package=emmeans

Müller, K. (2017). here: A simpler way to find your files. https://CRAN.R-project.org/package=here

National Center for Health Statistics. (2018). Underlying cause of death, 1999-2018. CDC WONDER Online Database.

Nelson, B. D., Perlman, G., Klein, D. N., Kotov, R., \& Hajcak, G. (2016). Blunted Neural Response to Rewards as a Prospective Predictor of the Development of Depression in Adolescent Girls. American Journal of Psychiatry, 173(12), 1223-1230. https://doi.org/10.1176/appi.ajp.2016.15121524

Pizzagalli, D. A., Jahn, A. L., \& O’Shea, J. P. (2005). Toward an objective characterization of an anhedonic phenotype: A signal-detection approach. Biological Psychiatry, 57(4), 319-327. https://doi.org/10.1016/j.biopsych.2004.11.026

Proudfit, G. H. (2015). The reward positivity: From basic research on reward to a biomarker for depression: The reward positivity. Psychophysiology, 52(4), 449-459. https://doi.org/10.1111/psyp.12370

R Core Team. (2018). R: A language and environment for statistical computing. $\mathrm{R}$ Foundation for Statistical Computing. https://www.r-project.org/

Revelle, W. (2018). psych: Procedures for psychological, psychometric, and personality research. https://CRAN.R-project.org/package=psych

Singmann, H., Bolker, B., Westfall, J., \& Aust, F. (2019). afex: Analysis of factorial experiments. https://CRAN.R-project.org/package=afex

Tsypes, A., Owens, M., \& Gibb, B. E. (2019). Blunted Neural Reward Responsiveness in Children With Recent Suicidal Ideation. Clinical Psychological Science, 216770261985634. https://doi.org/10.1177/2167702619856341

Wickham, H., Averick, M., Bryan, J., Chang, W., McGowan, L. D., François, R., Grolemund, G., Hayes, A., Henry, L., Hester, J., Kuhn, M., Pedersen, T. L., Miller, E., Bache, S. M., Müller, K., Ooms, J., Robinson, D., Seidel, D. P., Spinu, V., ... Yutani, H. (2019). 
Welcome to the tidyverse. Journal of Open Source Software, 4(43), 1686.

https://doi.org/10.21105/joss.01686

Wickham, H., \& Miller, E. (2019). haven: Import and export "SPSS", "stata” and "SAS" files. https://CRAN.R-project.org/package=haven 
Table 1

Means and Standard Deviations of Study Variables

Study 1

\begin{tabular}{ccc}
\hline Variable & No SI & Recent SI \\
\hline & $M(S D)$ & $M(S D)$ \\
\hline Wins & $18.20(8.18)$ & $16.09(7.91)$ \\
Losses & $13.95(7.91)$ & $12.18(7.44)$ \\
\hline$\Delta$ RewP & $4.26(6.03)$ & $3.91(5.20)$ \\
\hline
\end{tabular}

Study 2

\begin{tabular}{ccccc}
\hline Variable & No SI & Wave 1 SI Only & Wave 2 SI Only & Wave 1 \& 2 SI \\
\hline & $M(S D)$ & $M(S D)$ & $M(S D)$ & $M(S D)$ \\
\hline Wins (T1) & $3.36(6.52)$ & $2.35(4.55)$ & $3.63(6.68)$ & $4.33(6.23)$ \\
Losses (T1) & $-0.81(5.69)$ & $-1.70(5.14)$ & $2.64(8.28)$ & $-1.76(6.38)$ \\
$\Delta$ RewP (T1) & $4.17(6.02)$ & $4.05(5.28)$ & $0.99(5.06)$ & $6.09(6.03)$ \\
Wins (T2) & $6.65(6.03)$ & $6.50(7.30)$ & $6.68(4.81)$ & $6.42(4.34)$ \\
Losses (T2) & $2.76(5.70)$ & $2.11(5.72)$ & $3.46(4.55)$ & $0.85(3.99)$ \\
\hline$\Delta$ RewP (T2) & $3.90(6.75)$ & $4.39(6.87)$ & $3.22(5.69)$ & $5.56(4.78)$ \\
\hline
\end{tabular}

Note. SI $=$ Suicidal ideation, $\mathrm{T} 1=$ Time $1, \mathrm{~T} 2=$ Time $2, \Delta$ RewP $=$ Reward positivity (wins losses). 


\section{Figure 1}

Study 1 Stimulus-Locked Event-Related Potentials

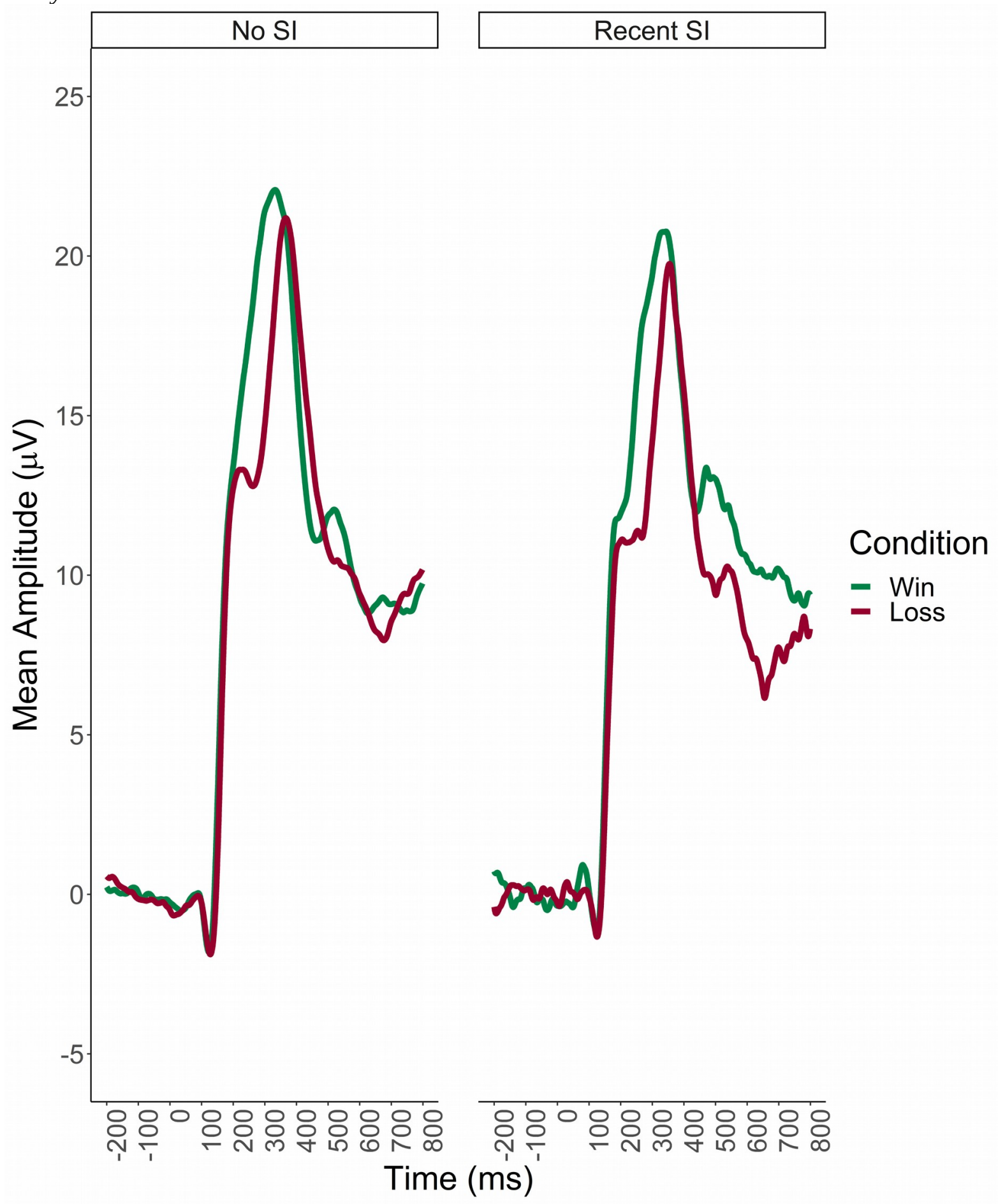

Note. Waveforms represent event-related potentials to wins and losses at $\mathrm{Cz}$ without conducting temporosptatial PCA. 


\section{Figure 2}

Study $1 \Delta$ RewP of Children and Adolescents With and Without Recent SI

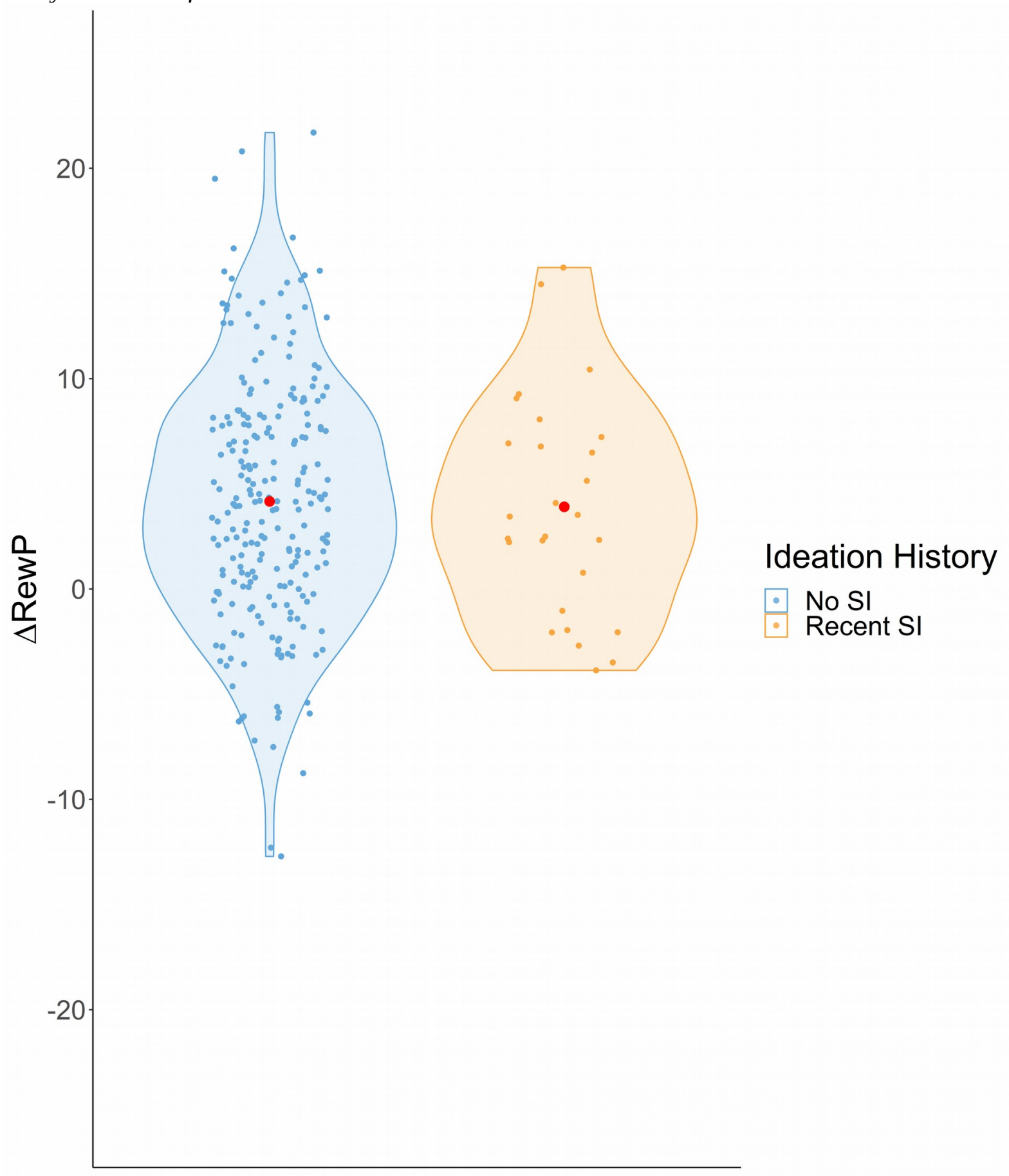

Note. Red point represents mean $\Delta$ RewP in each group. 


\section{Figure 3}

Study $2 \Delta$ RewP of Children and Adolescents Across Wave 1 and Wave 2

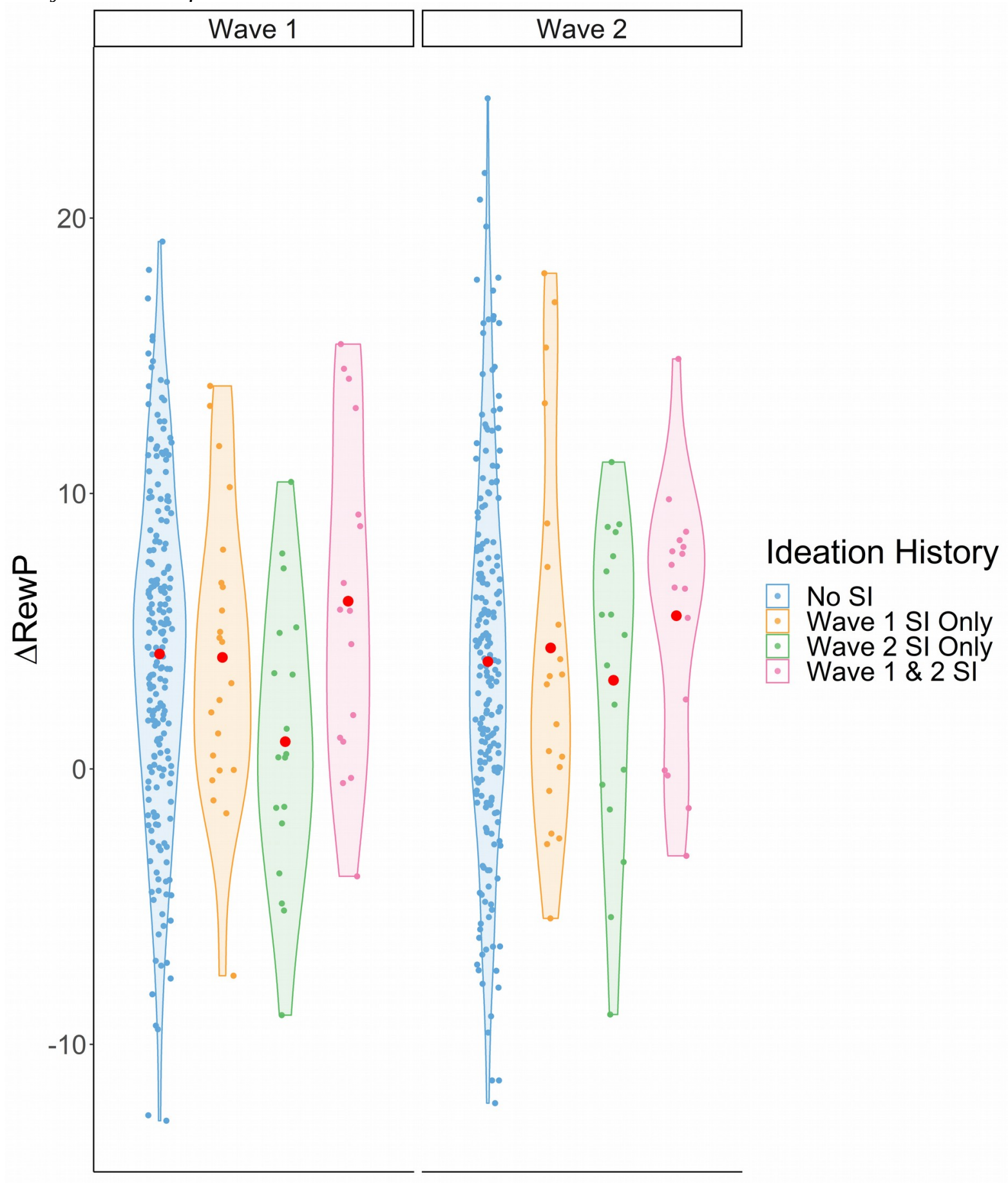

Note. Red points represent mean $\Delta$ RewP across groups. 